\title{
Editorial
}

\section{The Next Frontier of Post-Translational Modifications}

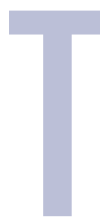

he human genome encodes approximately 20,000 protein-encoding genes. ${ }^{1}$ This complexity is increased by several orders of magnitude by including the number of splice variants and diverse posttranslational modifications (PTMs), which likely lead to millions of different functional members of the human proteome. Hundreds of unique PTMs have been described, although some are more thoroughly understood due to the availability of tools and reagents. The most commonly studied PTMs include phosphorylation, ubiquitylation, glycosylation, acetylation, lipidation, oxidation, and proteolysis. These distinct modifications function to rapidly switch proteins to different active or inactive conformations, change polarity or hydrophobicity to affect localization, recruit new interaction partners, or target degradation. Most PTMs occur at polar and charged amino acids, including serine, threonine, asparagine, cysteine, lysine, and arginine. Even after decades of studying phosphorlyation, our knowledge of the dynamics and diversity of PTM continues to expand with the development of new analytical methods and tools. PTMs are clearly required for the activation, inhibition, and degradation of nearly all proteins. Undoubtedly, the complexity of post-translational regulation makes the task of translating genomic information to the biological function of proteins daunting. In this issue, six reviews are presented that outline progress in understanding the diversity and regulation of distinct posttranslational events.

Metabolic labeling with radioactive phosphate, acetate, sugars, and lipids provided the first evidence of the breadth of unique modifications in the proteome. Later, PTM-specific antibodies greatly enhanced the ability to rapidly analyze specific modifications, especially those that are sufficiently antigenic. Recent advances in mass spectrometry have provided the first proteome-wide view many PTMs. These experiments are massively more sensitive after selective enrichment, which reduces abundant unmodified peptides. Such studies have identified tens of thousands of phosphorylation sites. ${ }^{2}$ Such studies

\footnotetext{
Published online 5 August 2013 in Wiley Online Library (wileyonlinelibrary.com). DOI 10.1002/bip.22370

() 2013 Wiley Periodicals, Inc.
}

demonstrate the broad impact of PTMs, but also the requirement for sensitive enrichment and detection methods.

This issue of Biopolymers contains six reviews on methods to study PTMs. This includes a focused review by Slade, et.al. on arginine modifications, including methylation, ADP-ribosylation, phosphorylation, and deimidation. ${ }^{3}$ This review discusses the enzymes that regulate these modifications, biological implications, and emerging methodologies to annotate sites of modification. Weller, et al. present an update on chemical methods for understanding the complex language of lysine ubiquitylation, ${ }^{4}$ specifically focused on semi-synthetic methods for controlled ubiquitylation of proteins for biochemical and structural studies. This review highlights additional functional and structural roles for ubiquitylation beyond targeted degradation.

Next, there are three reviews focused on cysteine modifications. Cysteine is a unique amino acid is both redox reactive and nucleophilic. This leads to an array of both oxidative and enzymatic modifications. Shannon and Weerapana review rare cysteine PTMs, including methylation, phosphorylation, and ubiquitylation. ${ }^{5}$ They discuss the biological significance of the poorly characterized and scarce modifications that occur in both mammals and bacteria. Pan and Carroll review recent advances in understanding cysteine sulfenylation, and the merging functional role in of oxidative regulation of key signaling pathways. ${ }^{6}$ This includes the development of new methods to selectively tag and enrich sulfenylation for biochemical analysis and mass spectrometry annotation. In addition, Majmudar and Martin review recent advances in methods to study native S-nitrosylation, ${ }^{7}$ the product of enzymatically generated reactive nitrogen species. This includes discussion on the mechanism of S-nitrosylation, and emerging methods to annotate and characterize this labile modification.

Finally, Hertel and Zhang discuss recent advances developing genetically-encoded fluorescent reporters for studying the spatial and temporal dynamics of PTMs. ${ }^{8}$ This includes recent tools to visualize the activity of kinases, phosphatases, and acetyl-transferases, methyl-transferases, and proteases. These tools provide critical information on the spatial 
regulation and dynamics of PTMs in intact, living cells and organisms.

Overall, these reviews present an update on methods, tools, and new avenues of exploring post-translation regulation of the proteome. Clearly, the next frontier is to continue developing new enrichment methods and analytical tools to understand the dynamics, stoichiometry, and functional interplay of the breadth of PTMs in the proteome.

BRENT R. Martin

Department of Chemistry, University of Michigan Ann Arbor, MI 48019

\section{REFERENCES}

1. International Human Genome Sequencing Consortium. Nature 2004, 431, 931-945.

2. Huttlin, E. L.; Jedrychowski, M. P.; Elias, J. E.; Goswami, T.; Rad, R.; Beausoleil, S. A.; Villen, J.; Haas, W.; Sowa, M. E.; Gygi, S. P. Cell 2010, 143, 1174-1189.

3. Slade, D. J.; Subramanian, V.; Fuhrmann, J.; Thompson, P. R. Biopolymers 2014, 101, 133-143.

4. Weller, C. E.; Pilkerton, M. E.; Chatterjee, C. Biopolymers 2014, 101, 144-155.

5. Shannon, D. A.; Weerapana, E. Biopolymers 2014, 101, 156-164.

6. Pan, J.; Carroll, K. S. Biopolymers 2014, 101, 165-172.

7. Majmudar, J. D.; Martin, B. R. Biopolymers 2014, 101, 173-179.

8. Hertel, F.; Zhang, J. Biopolymers 2014, 101, 180-187. 